\title{
Charge density wave with anomalous temperature dependence in $\mathrm{UPt}_{2} \mathrm{Si}_{2}$
}

\author{
Jooseop Lee $\odot,{ }^{1,2}$ Karel Prokeš, ${ }^{3}$ Sohee Park, ${ }^{1}$ Igor Zaliznyak $\odot,{ }^{4}$ Sachith Dissanayake, ${ }^{5}$ Masaaki Matsuda, ${ }^{5}$ \\ Matthias Frontzek ${ }^{\circ},{ }^{5}$ Stanislav Stoupin, ${ }^{2}$ Greta L. Chappell, ${ }^{6,7}$ Ryan E. Baumbach, ${ }^{6,7}$ Changwon Park, ${ }^{1}$ John A. Mydosh, ${ }^{8}$ \\ Garrett E. Granroth, ${ }^{5}$ and Jacob P. C. Ruff ${ }^{2}$ \\ ${ }^{1}$ CALDES, Institute for Basic Science, Pohang 37673, Republic of Korea \\ ${ }^{2}$ CHESS, Cornell University, Ithaca, New York 14853, USA \\ ${ }^{3}$ Helmholtz-Zentrum Berlin für Materialien und Energie, Berlin 14109, Germany \\ ${ }^{4}$ CMPMSD, Brookhaven National Laboratory, Upton, New York 11973, USA \\ ${ }^{5}$ Neutron Scattering Division, Oak Ridge National Laboratory, Oak Ridge, Tennessee 37831, USA \\ ${ }^{6}$ National High Magnetic Field Laboratory, Florida State University, Tallahassee, Florida 32310, USA \\ ${ }^{7}$ Department of Physics, Florida State University, Tallahassee, Florida 32306, USA \\ ${ }^{8}$ Institute Lorentz and Kamerlingh Onnes Laboratory, Leiden University, Leiden 2300 RA, The Netherlands
}

(Received 20 September 2019; revised 29 June 2020; accepted 29 June 2020; published 9 July 2020; corrected 1 October 2020)

\begin{abstract}
Using single-crystal neutron and x-ray diffraction, we discovered a charge density wave (CDW) below $320 \mathrm{~K}$, which accounts for the long-sought origin of the heat capacity and resistivity anomalies in $\mathrm{UPt}_{2} \mathrm{Si}_{2}$. The modulation wave vector, $\boldsymbol{Q}_{\text {mod }}$, is intriguingly similar to the incommensurate wave vector of $\mathrm{URu}_{2} \mathrm{Si}_{2}$. $\boldsymbol{Q}_{\text {mod }}$ shows an unusual temperature dependence, shifting from commensurate to incommensurate position upon cooling and becoming locked at aproximately $(0.4200)$ near $180 \mathrm{~K}$. Bulk measurements indicate a crossover toward a correlated coherent state around the same temperature, suggesting an interplay between the CDW and Kondo-lattice-like coherence before coexisting antiferromagnetic order sets in at $T_{\mathrm{N}}=35 \mathrm{~K}$.
\end{abstract}

DOI: 10.1103/PhysRevB.102.041112

In strongly correlated $5 f$-electron systems, interactions with comparable strength compete due to the extended nature of the wave functions. This competition leads to a rich variety of exotic states, which can hardly be understood with conventional models from $d$ - or $4 f$-electron physics [1]. In metallic U-based heavy fermion compounds with strong hybridization effects of the surrounding ligands, an exceptional coexistence of unusual phases occurs as, for example, in the hidden order superconductor $\mathrm{URu}_{2} \mathrm{Si}_{2}$. The nature of the "hidden order" parameter responsible for the heat capacity anomaly is still debated, more than 30 years after its discovery [2].

$\mathrm{UPt}_{2} \mathrm{Si}_{2}$ is a closely related intermetallic compound of the $\mathrm{UT}_{2} M_{2}(T=\operatorname{transition~metal} ; M=\mathrm{Si}$ or $\mathrm{Ge})$ family whose Pt$5 d$ electrons hybridize with the U- $5 f$ states. $\mathrm{UPt}_{2} \mathrm{Si}_{2}$ adopts the $\mathrm{CaBe}_{2} \mathrm{Ge}_{2}$ crystal structure and orders magnetically at $T_{\mathrm{N}}=35 \mathrm{~K}$ with wave vector $\boldsymbol{Q}_{\mathrm{M}}=(100)$, where ferromagnetic $a b$ planes are stacked antiferromagnetically (AFM) along the $c$ axis, with a large ordered magnetic moment of $\approx 2 \mu_{B}$ [3-5]. Consequently, $\mathrm{UPt}_{2} \mathrm{Si}_{2}$ has long been considered a rare example of a uranium intermetallic compound with localized $5 f$ electrons where magnetism can be explained within a simple crystal-field level scheme [4]. Several recent studies [6-9], however, questioned the degree of electron localization in this system. High-field measurements suggest that phase transitions under applied magnetic field should be understood in terms of Fermi surface effects [6]. This approach has been further supported by a density functional theory (DFT) calculation which favors a scenario where the $5 f$ electrons are mostly itinerant [7]. A recent inelastic neutron-scattering study reveals a dual nature, both itinerant and local, in the magnetic excitation spectrum of $\mathrm{UPt}_{2} \mathrm{Si}_{2}$ at low temperatures [9].

The crystal structure of $\mathrm{UPt}_{2} \mathrm{Si}_{2}$ and its relationship with electronic properties have been another important longstanding controversy [10-12]. The x-ray-diffraction measurements found large anisotropic thermal factors on one of the two Pt and Si sites, which were interpreted as an indication of strong crystallographic disorder [11]. This purported disorder was thought to be responsible for the highly anisotropic resistivity with Anderson localization along the $c$ axis.

The origin and the nature of the disorder in this moderately heavy fermion system, however, has long been open to questions. Most $\mathrm{UT}_{2} \mathrm{M}_{2}$ compounds crystallize into the $\mathrm{ThCr}_{2} \mathrm{Si}_{2}-$ type structure (space group $14 \mathrm{mmm}$ ) and only a few, including $\mathrm{UPt}_{2} \mathrm{Si}_{2}$, have been reported to adopt the primitive $\mathrm{CaBe}_{2} \mathrm{Ge}_{2}-$ type structure. Therefore, when a specific heat anomaly and a concomitant cusp in the electrical resistivity around room temperature were found [13], it was anticipated that there could be a structural transition between the two closely related crystal structures as in $\mathrm{UCo}_{2} \mathrm{Ge}_{2}$ [14], leading to disorder. However, $\mathrm{x}$-ray-diffraction measurements on a polycrystalline sample did not find any indication of a structural change. Moreover, a comparative electrical transport study of asgrown and annealed samples showed that the effect of the presumed disorder does not depend on synthesis conditions or impurity level [13].

Here, we report single-crystal neutron and $\mathrm{x}$-raydiffraction measurements, which reveal the discovery of a periodic lattice modulation that most likely results from a charge density wave $(\mathrm{CDW})$ which develops in $\mathrm{UPt}_{2} \mathrm{Si}_{2}$ below $T_{s} \approx$ $320 \mathrm{~K}$. The appearance of the CDW is responsible for the heat 

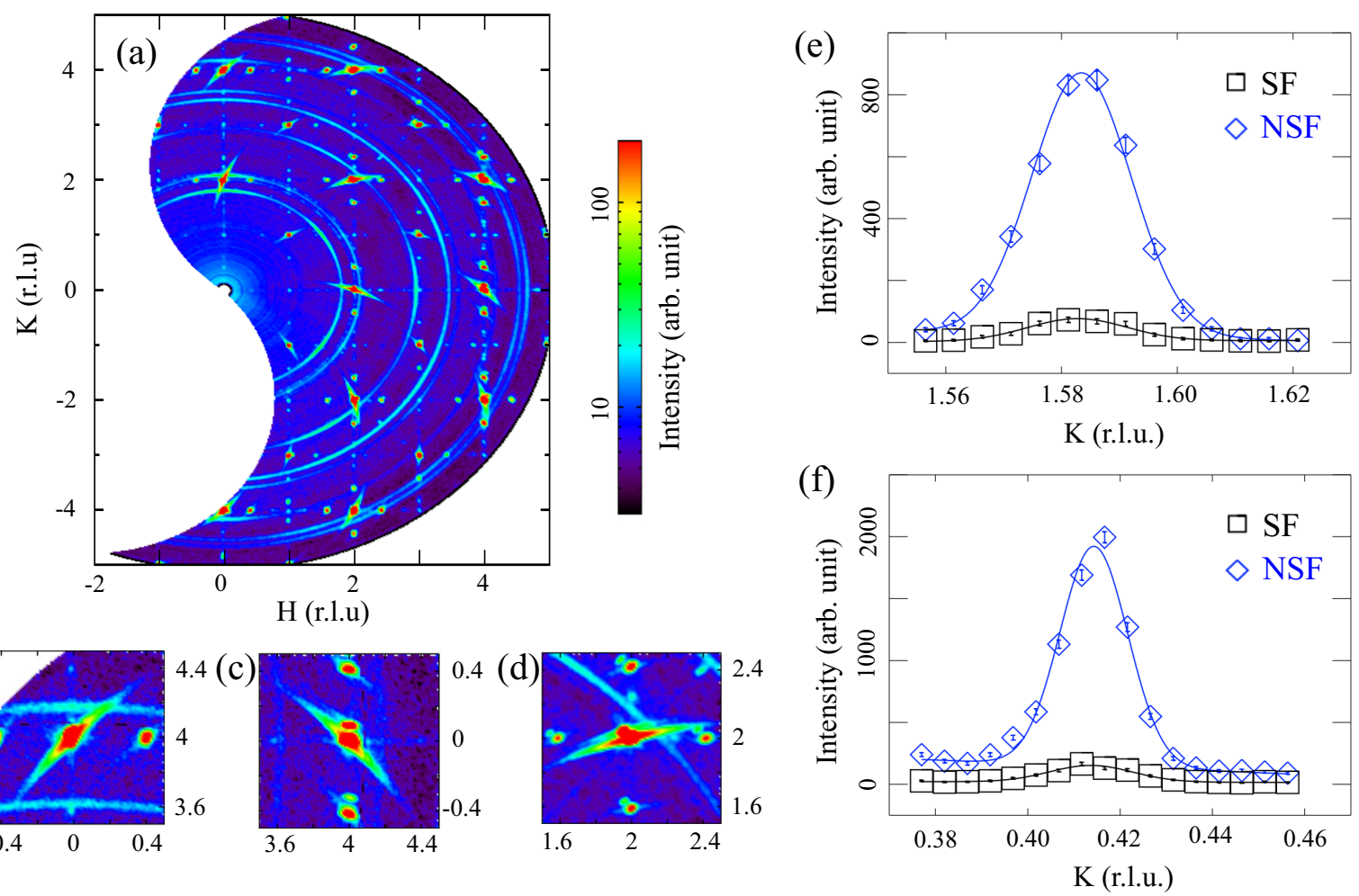

FIG. 1. Neutron diffraction measured at $50 \mathrm{~K}$ with WAND. (a) A full map in the $\left(\begin{array}{l}H \\ K\end{array}\right)$ plane and zoomed-in views around (b) ( 040$)$, (c) $\left(\begin{array}{lll}4 & 0 & 0\end{array}\right)$, and (d) (2 20 ) showing peak extinction rules that reveal the polarization of atomic displacements. SF and NSF intensities from PTAX measured at $50 \mathrm{~K}$ at (e) $\mathbf{Q}=\left(\begin{array}{lll}2 & 0.42 & 0\end{array}\right)$ and (f) (2 1.580$)$, where solid lines are fit to a Gaussian.

capacity and electrical resistivity anomalies and resolves the controversy surrounding the putative "disorder." The CDW wave vector $\left(\begin{array}{lll}\tau & 0 & 0\end{array}\right)$ shows an interesting temperature dependence, shifting up on cooling from a commensurate value of $\tau=0.40$ just below $T_{s}$ to lock-in value at $\tau \approx 0.42$ around $180 \mathrm{~K}$. Around the same temperature, electrical transport data indicates a crossover from a local to a coherent electronic state on cooling, suggesting an interplay between the CDW and Kondo-lattice physics in this system. This unusual type of CDW that involves strongly correlated U-5 $f$ states appears at relatively high temperatures and coexists at low temperatures with an antiferromagnetic order. Intriguingly, the incommensurate CDW modulation wave vector, $\boldsymbol{Q}_{\text {mod }}=$ ( $\tau 00)$ with $\tau \approx 0.4$, resembles the incommensurate wave vector of $\mathrm{URu}_{2} \mathrm{Si}_{2}$ where the softening of magnetic excitation is observed $[15,16]$, implying that similar underlying physics may be at play.

Neutron-diffraction measurements were carried out at the wide angle neutron diffractometer (WAND) and HB1 polarized triple-axis spectrometer (PTAX) at the High Flux Isotope Reactor, ORNL and E4 two-axis diffractometer [17] at the Helmholtz-Zentrum Berlin. For the PTAX measurement, the neutron spin was aligned parallel to wave-vector transfer, $\boldsymbol{q}$, so that nuclear and magnetic scattering were measured in nonspin-flip (NSF) and spin-flip (SF) channel, respectively. The flipping ratio of PTAX was approximately 15 . Synchrotron $\mathrm{x}$ ray experiments were performed at the A1 beamline at Cornell High Energy Synchrotron Source (CHESS). We carried out DFT calculations via the generalized gradient approximation using the Perdew-Burke-Ernzerhof functional [18], as implemented in the VASP package $[19,20]$.

Figure 1(a) presents single-crystal neutron -diffraction data obtained at the WAND neutron diffractometer at $T=50 \mathrm{~K}$, which is above $T_{\mathrm{N}}=35 \mathrm{~K}$ but well below the reported heat capacity anomaly temperature of $\approx 320 \mathrm{~K}$ [13]. We observe a pattern of satellite peaks offset from the original $P 4 / \mathrm{nmm}$ Bragg reflections by a wave vector $\boldsymbol{Q}_{\text {mod }}=\left(\begin{array}{lll}\tau & 0 & 0\end{array}\right)$ or $\left(\begin{array}{lll}0 & \tau & 0\end{array}\right)$, where $\tau \approx 0.42$. We investigated the origin of these superlattice reflections by performing neutron polarization analysis on the $\left(\begin{array}{llll}2 & 0.42 & 0\end{array}\right)$ and $\left(\begin{array}{lll}2 & 1.58 & 0\end{array}\right)$ satellite peaks at $50 \mathrm{~K}$ at HB1 triple-axis spectrometer as shown in Figs. 1(e) and 1(f), respectively. NSF scattering dominates the intensity of both reflections, confirming their nonmagnetic, charge/lattice origin. Our findings thus correct the previous reports on the atomic disorder [11], which in fact resulted from the misassignment of the observed changes of the lattice principal Bragg reflection intensities to disorder rather than lattice modulation induced by $\mathrm{CDW}$.

By virtue of the WAND diffraction patterns covering a wide reciprocal space area [Fig. 1(a)], we can clearly identify the polarization of the observed atomic displacements. As shown in Figs. 1(b) and 1(c), the intensities of longitudinal superlattice peaks, $\left(\begin{array}{lll}H \pm \tau & 0 & 0\end{array}\right)$, or, equivalently, $\left(\begin{array}{l}0 \pm \tau \\ 0\end{array}\right.$ 0 ), are absent, or very weak compared to those of transverse superlattice peaks, $( \pm \tau K 0)$ and $(H \pm \tau 0)$. For example, the integrated intensity of a longitudinal $(4.4200)$ peak is about 240 times smaller compared to the corresponding transverse (4 0.42 0) peak. For lattice Bragg peaks near the diagonal 


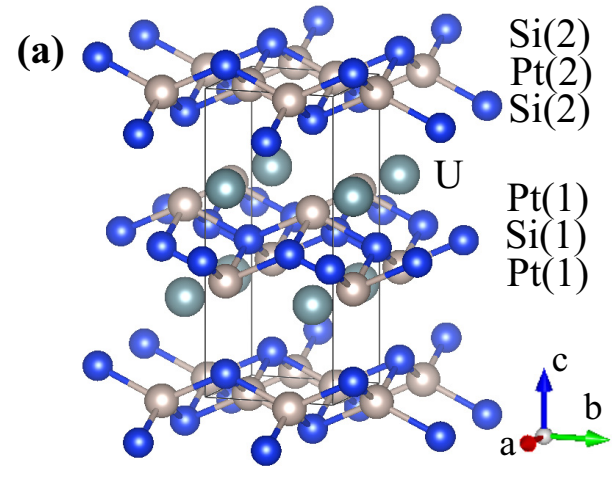

(b)

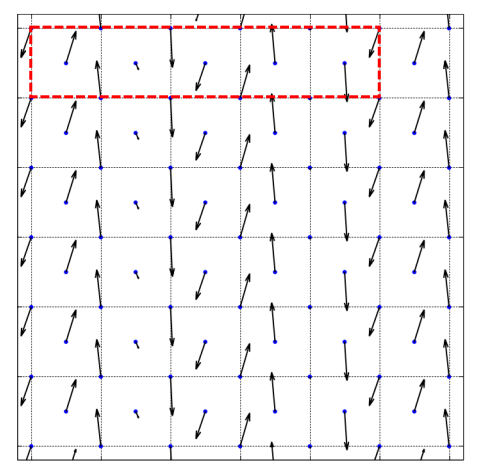

(c)

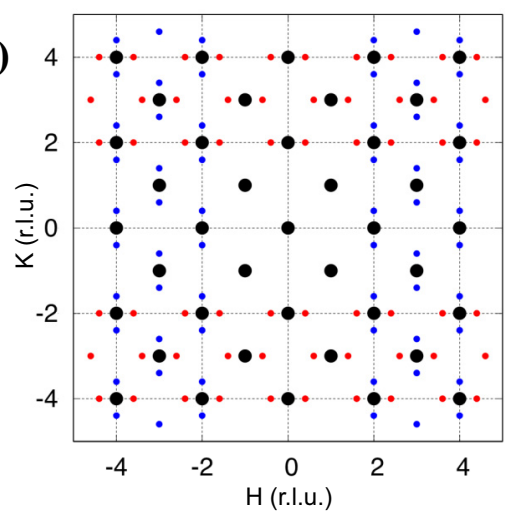

FIG. 2. (a) Crystal structure of $\mathrm{UPt}_{2} \mathrm{Si}_{2}$ where bonds between $\mathrm{Pt}$ and $\mathrm{Si}$ atoms illustrate two inequivalent $\mathrm{Pt}-\mathrm{Si}$ layers in the $\mathrm{CaBe} \mathrm{Ge}_{2}$ structure. The atomic labels follow the convention of [11], origin 2 of $P 4 / n m m$, which reported disorder in $\mathrm{UPt}_{2} \mathrm{Si}_{2}$. (b) Calculated displacements of $\operatorname{Pt}(2)$ atoms in the $a b$ plane within one domain. The red rectangle is a $5 \times 1 \times 1$ calculation supercell, where blue dots and black arrows represent the original position and 15 times of atomic displacement, respectively. (c) Simulated reflection pattern from the displaced Pt atoms. The radii of the dots are proportional to the squares of Fourier components while Bragg peaks are reduced by $30 \%$ for clarity.

$\left(\begin{array}{l}H \\ H\end{array}\right)$ direction we find all four satellite reflections with comparable intensity [Fig. 1(d)]. There exist weak reflections at $\left(\begin{array}{ll}H & K\end{array}\right)$ with $H+K=$ odd inconsistent with $P 4 / n m m$ symmetry, which, however, turns out to be due to an internal strain [5].

The intensity of a satellite peak at a momentum transfer $\boldsymbol{q}$ can be expressed as $[21,22]$

$$
I(\boldsymbol{q}) \approx\left|\sum_{v}\left(\boldsymbol{q} \cdot \boldsymbol{\epsilon}_{v}\right) f_{v}\left(\boldsymbol{q} \pm \boldsymbol{Q}_{\mathrm{mod}}\right)\right|^{2} \delta\left(\boldsymbol{q}-\boldsymbol{G} \pm \boldsymbol{Q}_{\mathrm{mod}}\right),
$$

e.g., using Taylor expansion of Jacobi-Anger identity. Here, $\boldsymbol{\epsilon}_{v}$ is the displacement of atom $v$ in the unit cell, $f_{v}$ is the partial structure factor of atom $v$, and $\boldsymbol{G}$ is the reciprocal lattice vector. The $\left(\boldsymbol{q} \cdot \boldsymbol{\epsilon}_{\nu}\right)$ prefactor in the satellite reflection intensity is responsible for the observed polarization dependence of the diffraction pattern, indicating that the atomic displacements, $\boldsymbol{\epsilon}_{\nu}$, in $\mathrm{UPt}_{2} \mathrm{Si}_{2}$ are mostly transverse, either along the $a$-axis direction for $\boldsymbol{Q}_{\text {mod }} \approx(00.40)$, or along the $b$-axis direction for $\boldsymbol{Q}_{\text {mod }} \approx\left(\begin{array}{lll}0.4 & 0 & 0\end{array}\right)$. While the observed pattern of superlattice reflections can be regarded as overlaid patterns from two $\boldsymbol{Q}$ domains, within our resolution we did not detect any clear sign of tetragonal symmetry breaking in the main Bragg reflections.

To understand the atomic modulation pattern in real space, we performed DFT calculation by relaxing the atomic positions in a $4 \times 1 \times 1,5 \times 1 \times 1$, and $6 \times 1 \times 1$ supercell. For the $5 \times 1 \times 1$, case, we find a reduction of the total energy by $8.6 \mathrm{meV}$ per unit cell with the presence of the CDW. This confirms the CDW to be unidirectional, that is, each $Q$ domain is described by a single wave vector. As shown in Fig. 2(a), there are two kinds of Pt-Si layers in $\mathrm{UPt}_{2} \mathrm{Si}_{2}$. In the $\mathrm{Si}(2)-\mathrm{Pt}(2)-\mathrm{Si}(2)$ layer, a square lattice of $\mathrm{Pt}$ atoms is sandwiched between $\mathrm{Si}$ atoms, while in the $\mathrm{Pt}(1)-\mathrm{Si}(1)$ $\operatorname{Pt}(1)$ layer the situation is inverted. Our calculation shows that about $90 \%$ of displacement is in the $a b$ plane of the $\mathrm{Si}(2)-\operatorname{Pt}(2)-\mathrm{Si}(2)$ layer, in agreement with previously reported putative disorder [11]. It is interesting that the lattice modulation of sandwiched metallic layers is quite ubiquitous [23] as in the Pt-based layered superconductor $\mathrm{LaPt}_{2} \mathrm{Si}_{2}$ [24-26]. It should be, however, noted that the period of the $\mathrm{LaPt}_{2} \mathrm{Si}_{2}$ superstructure is close to 3 unit cells compared to the $\approx 5$ unit cell periodicity in $\mathrm{UPt}_{2} \mathrm{Si}_{2}$. Here, we expect the strongly correlated $5 f$-electron states to participate in the bonding due to hybridization between $\mathrm{U}-5 f$ and Pt- $5 d$ states.

The calculated real space displacement pattern of the $\mathrm{Pt}(2)$ layer is shown in Fig. 2(b), where the dashed red rectangle represents the calculated supercell. When the CDW modulation wave vector is given along the $a$ axis, the atomic displacement is almost along the $b$ axis. Figure 2(c) shows the Fourier transform of the Pt atomic positions, which can well explain the polarization of the observed reflections. Black, red, and blue dots correspond to principal Bragg peaks, satellite peaks from the Fig. 2(b) structure, and its $90^{\circ}$-rotated domain, respectively.

Figure 3(a) presents the evolution of the superlattice Bragg peak at $\boldsymbol{q}=(2 \tau 0)$ with temperature. The intensity, Fig. 3(c), keeps increasing as the sample is cooled down, indicating gradual development of the superlattice modulation. Here, we find a second-order-like transition with a transition temperature $T_{\mathrm{s}}=319(8) \mathrm{K}$, consistent with the previously reported heat capacity and electrical resistivity anomaly temperature [13]. Fitting the temperature dependence of the order parameter to expression $I \propto\left(T_{c}-T\right)^{2 \beta}$, we obtain the critical exponent $\beta=0.39(10)$ which, within our accuracy, is consistent with both 3D universality and with mean-field behavior. Below $T_{\mathrm{N}}=35 \mathrm{~K}$, the $\mathrm{CDW}$ coexists with the antiferromagnetic order.

Surprisingly, as shown in Fig. 3(b), the satellite peak moves away from the nearly commensurate position of $\tau=0.400(5)$ observed around $300 \mathrm{~K}$ and becomes lockedin at an incommensurate value $0.418(2)$ below $\approx 180 \mathrm{~K}$ suggesting an unusual thermal evolution of the CDW. As the energy to lock-in to the commensurate lattice is a function of the CDW amplitude, which increases at low temperature, an opposite, commensurate to incommensurate lock-in of CDW upon cooling observed here is extremely rare $[27,28]$. Such a CDW wave-vector shift suggests that the 

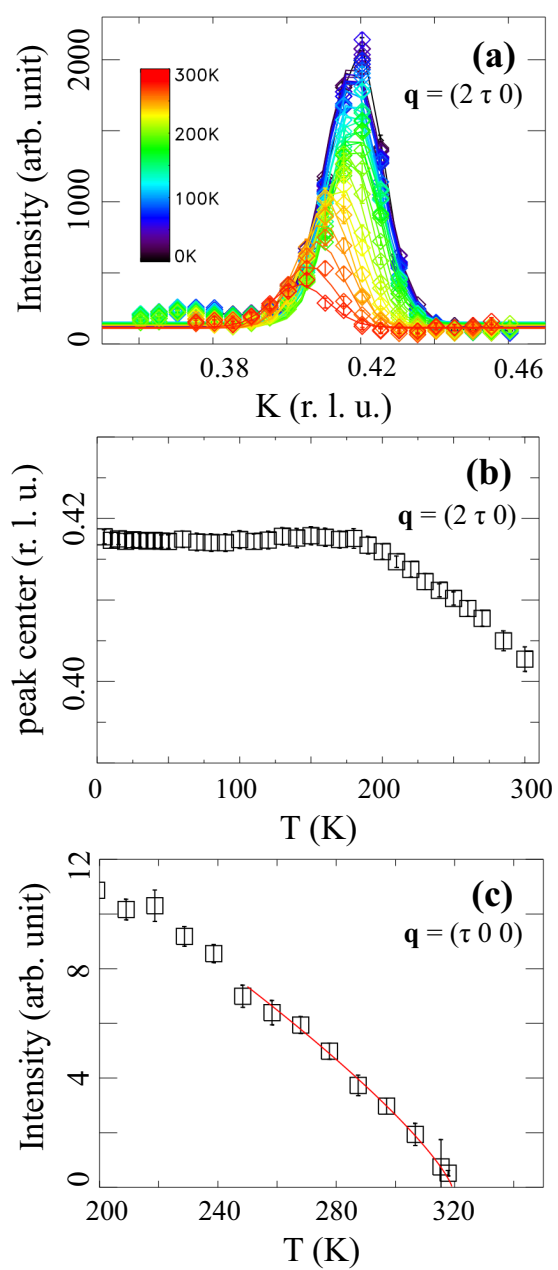

FIG. 3. (a) Temperature dependence of the $(2 \tau 0)$ superlattice peak. Solid lines are fit to Gaussian. (b) Temperature-dependent peak position of $\tau$, which locks in around $T_{\text {coh }}=180 \mathrm{~K}$. (c) Integrated intensity of ( $\left.\begin{array}{lll}\tau & 0 & 0\end{array}\right)$ measured up to $317 \mathrm{~K}$. Red solid line is the best fit to critical behavior described in the text. The data presented in panels (a) and (b) are measured from 4 to $300 \mathrm{~K}$ at PTAX in NSF configuration and therefore are of charge/lattice origin in nature. The unpolarized data in (c) were measured at E4.

electronic structure of uranium plays an important role at low temperatures.

Previously reported bulk measurements indicate that around the same temperature the $a$-axis resistivity shows a broad maximum [29,30] and the magnetic susceptibility begins to deviate from the Curie-Weiss law [4]. These observations suggest an onset of a "Kondo-lattice-type" coherence that marks a crossover from local moments and weakly correlated electrons to correlated, heavy-electron behavior. Therefore, the unusual gradual change of $\boldsymbol{Q}_{\text {mod }}$ from commensurate to incommensurate is likely related to a transition from local moments towards a system with a dual character of the $5 f$ electrons [9] facilitated by the $5 f-5 d$ hybridization and indicates an interplay between CDW and Kondo-lattice-like coherence in $\mathrm{UPt}_{2} \mathrm{Si}_{2}$. While there are a few systems that show the mere coexistence of CDW and Kondo states [1,31,32], we suggest $\mathrm{UPt}_{2} \mathrm{Si}_{2}$ as the first example of coupling between CDW and Kondo-lattice coherence effects.
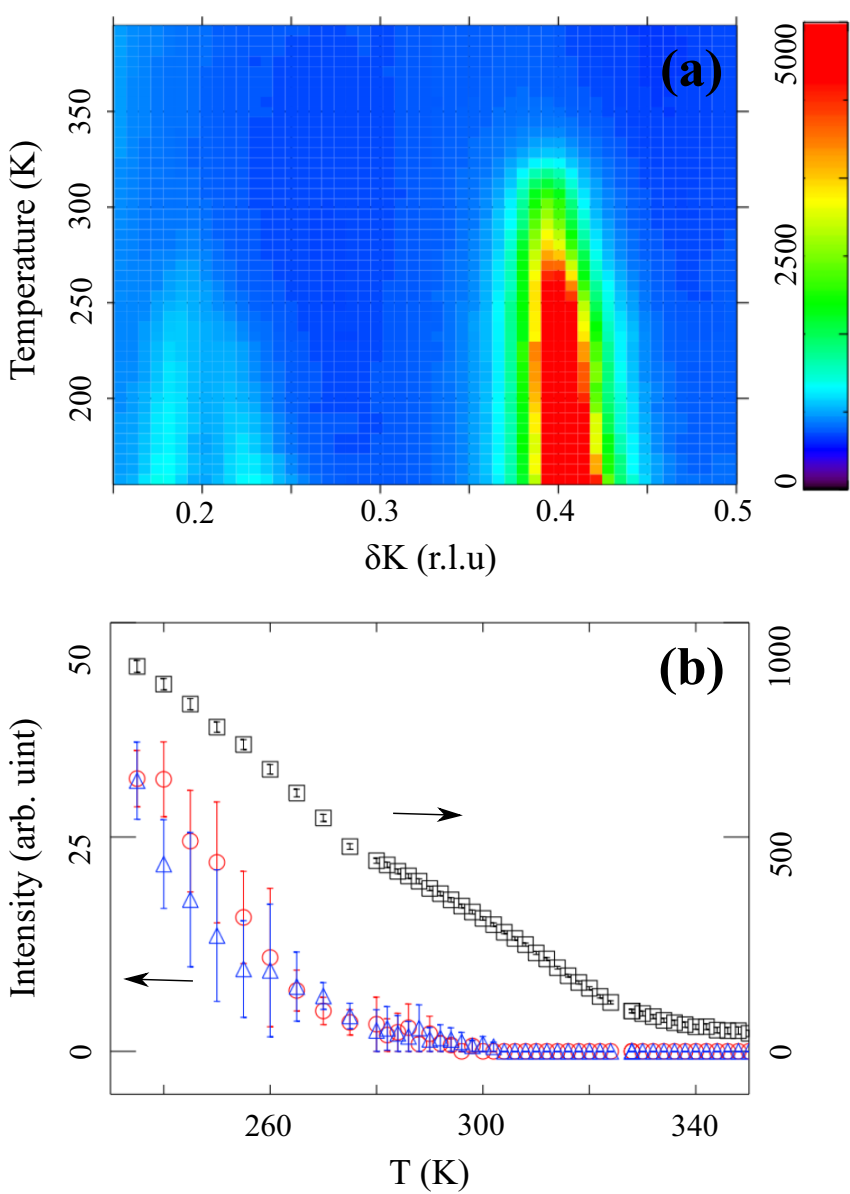

FIG. 4. Temperature dependence of the nonresonant synchrotron x-ray-diffraction pattern measured using the synchrotron x-ray at A1, CHESS. (a) Temperature dependence of superlattice harmonics around $q=(43-\delta K 2)$. (b) Temperature dependence of integrated intensities of principal harmonic (black squares), second harmonic (red circles), and third harmonic (blue triangles). The axis for the principal harmonic is shown on the right.

The nature of the CDW has been investigated in more detail with a synchrotron x-ray-diffraction measurement. A careful inspection of the diffraction pattern reveals that besides the first harmonic superlattice reflections with $\boldsymbol{Q}_{\text {mod }}$, there are also weak superlattice reflections indexable as the second and the third harmonics located around $q=(43-\delta K 2)$, Fig. 4(a). At high temperature, weak second and third harmonics nearly overlap at $q=(43.82)$ as higher harmonics are found at $\left(43+2 \boldsymbol{Q}_{\text {mod }} 2\right)$ and $\left(45-3 \boldsymbol{Q}_{\text {mod }} 2\right)$, while $\boldsymbol{Q}_{\text {mod }}$ is commensurate with $\tau=0.4$. As the temperature decreases, the second and third harmonic peaks get stronger in intensity and split apart as the incommensurability increases. Below $T_{\text {coh }}$, their positions lock-in at the corresponding incommensurate values, $2 \tau$ and $3 \tau$, confirming their higher harmonics origin.

In Fig. 4(b), we show the temperature dependence of the integrated intensities of the first three harmonics. The onset of the principal harmonics at $T_{\mathrm{s}}$ precedes a long tail of critical fluctuation precursor to the CDW at $T>T_{\mathrm{s}}$, which is emphasized in an energy-integrating measurement without an analyzer. There is also a hint of a second phase transition around $270 \mathrm{~K}$, where the intensities of higher harmonics begin 
to increase, suggesting that the CDW order parameter becomes two-component, with two displacement polarizations. The existence of higher harmonics indicates that the atomic CDW modulation is not purely sinusoidal, as is also noted in DFT calculation.

It is provocative to compare $\mathrm{UPt}_{2} \mathrm{Si}_{2}$ with $\mathrm{URu}_{2} \mathrm{Si}_{2}$. While these systems seem to be very different at first glance, one with mainly itinerant and the other with mainly local $5 f$ electron states, they indeed share many common features. They have closely related crystal structures and the same magnetic structures although the magnetic order in $\mathrm{URu}_{2} \mathrm{Si}_{2}$ seems to hover in the background, ready to appear under pressure, dilution, or magnetic field $[33,34]$. Thermoelectric measurements [35] suggest that both systems show Fermi surface reconstruction with a partial gap opening in hidden order or antiferromagnetic states, respectively [36].

Here, we find that both systems have two similar important wave vectors at play [37]. In $\mathrm{URu}_{2} \mathrm{Si}_{2}$, strong and coherent magnetic excitations are observed around the antiferromagnetic wave vector, $\boldsymbol{Q}_{0}=\left(\begin{array}{lll}1 & 0 & 0\end{array}\right)$, and the incommensurate wave vector, $\boldsymbol{Q}_{1} \approx(1 \pm 0.400)$ [38,39], whose origin is commonly believed to be Fermi surface nesting. $\mathrm{UPt}_{2} \mathrm{Si}_{2}$, as we find here, develops a CDW with $\boldsymbol{Q}_{\text {mod }}=\left(\begin{array}{lll}0.4 & 0 & 0\end{array}\right)$ and antiferromagnetic order with $\boldsymbol{Q}_{\mathrm{M}}=\left(\begin{array}{lll}1 & 0 & 0\end{array}\right)$. Very recent scanning tunneling microscope measurement shows a moiré pattern in $\mathrm{URu}_{2} \mathrm{Si}_{2}$ possibly from a CDW related to $\boldsymbol{Q}_{1}$ [40]. It is tempting to suggest that similar wave vectors characterizing the respective ground states in $\mathrm{URu}_{2} \mathrm{Si}_{2}$ and $\mathrm{UPt}_{2} \mathrm{Si}_{2}$ might be more than just a pure coincidence, demanding further attention.

In conclusion, our diffraction experiments demonstrate that a CDW appears near room temperature in a strongly correlated U-based intermetallic compound, $\mathrm{UPt}_{2} \mathrm{Si}_{2}$. In the ground state, this CDW coexists with an AFM order, which appears at a much lower temperature. Higherharmonic $\mathrm{CDW}$ reflections, which arise on cooling below $\approx 270 \mathrm{~K}$ indicate nonsinusoidal lattice modulation that is also supported by DFT calculations. Furthermore, the CDW wave vector exhibits unique temperature dependence. The commensurate-incommensurate transition on cooling and lock-in near the coherence temperature challenges our current understanding of CDW phenomena and requires taking into account the $5 f$-ligand hybridization. This suggests Kondo-lattice-type physics and the dual nature of U-5 $f$ electrons at play in $\mathrm{UPt}_{2} \mathrm{Si}_{2}$. The similarity of wave vectors characterizing electronic order in the two seemingly different systems, $\mathrm{URu}_{2} \mathrm{Si}_{2}$ and $\mathrm{UPt}_{2} \mathrm{Si}_{2}$, requires further studies, which might shed light on our understanding of the hidden order phase and unconventional superconductivity.

We thank K.-B. Lee and D. Y. Kim for helpful discussion. The sample synthesis was supported by Stefan Süllow whose work has been supported by the DFG under Contract No. SU229/1. Work at Brookhaven National Laboratory was supported by Office of Basic Energy Sciences (BES), Division of Materials Sciences and Engineering, US Department of Energy (DOE), under Contract No. DE-SC0012704. A portion of this research used resources at the High Flux Isotope Reactor, a DOE Office of Science User Facility operated by the Oak Ridge National Laboratory. Research conducted at CHESS is supported by the National Science Foundation via Awards No. DMR-1332208 and No. DMR-1829070.
[1] G. R. Stewart, Rev. Mod. Phys. 73, 797 (2001).

[2] J. A. Mydosh and P. M. Oppeneer, Rev. Mod. Phys. 83, 1301 (2011).

[3] R. A. Steeman, E. Frikkee, S. A. M. Mentink, A. A. Menovsky, G. J. Nieuwenhuys, and J. A. Mydosh, J. Phys.: Condens. Matter 2, 4059 (1990).

[4] G. J. Nieuwenhuys, Phys. Rev. B 35, 5260 (1987).

[5] K. Prokeš, O. Fabelo, S. Süllow, J. Lee, J. A. Mydosh, Z. Kristallogr (2020), doi:10.1515/zkri-2020-0018.

[6] D. Schulze Grachtrup, M. Bleckmann, B. Willenberg, S. Süllow, M. Bartkowiak, Y. Skourski, H. Rakoto, I. Sheikin, and J. A. Mydosh, Phys. Rev. B 85, 054410 (2012).

[7] S. Elgazzar, J. Rusz, P. M. Oppeneer, and J. A. Mydosh, Phys. Rev. B 86, 075104 (2012).

[8] D. S. Grachtrup, N. Steinki, S. Süllow, Z. Cakir, G. Zwicknagl, Y. Krupko, I. Sheikin, M. Jaime, and J. A. Mydosh, Phys. Rev. B 95, 134422 (2017).

[9] J. Lee, M. Matsuda, J. A. Mydosh, I. Zaliznyak, A. I. Kolesnikov, S. Süllow, J. P. C. Ruff, and G. E. Granroth, Phys. Rev. Lett. 121, 057201 (2018).

[10] A. Otop, F. J. Litterst, R. W. A. Hendrikx, J. A. Mydosh, and S. Süllow, J. Appl. Phys. 95, 6702 (2004).

[11] S. Süllow, A. Otop, A. Loose, J. Klenke, O. Prokhnenko, R. Feyerherm, R. W. A. Hendrikx, J. A. Mydosh, and H. Amitsuka, J. Phys. Soc. Jpn. 77, 024708 (2008).
[12] S. Süllow, I. Maksimov, A. Otop, F. J. Litterst, A. Perucchi, L. Degiorgi, and J. A. Mydosh, Phys. Rev. Lett. 93, 266602 (2004).

[13] M. Bleckmann, A. Otop, S. Süllow, R. Feyerherm, J. Klenke, A. Loose, R. W. A. Hendrikx, J. A. Mydosh, and H. Amitsuka, J. Magn. Magn. Mater. 322, 2447 (2010).

[14] T. Endstra, G. J. Nieuwenhuys, A. A. Menovsky, and J. A. Mydosh, J. Appl. Phys. 69, 4816 (1991).

[15] S. Elgazzar, J. Rusz, M. Amft, P. M. Oppeneer, and J. A. Mydosh, Nat. Mater. 8, 337 (2009).

[16] C. Bareille, F. L. Boariu, H. Schwab, P. Lejay, F. Reinert, and A. F. Santander-Syro, Nat. Commun. 5, 4326 (2014).

[17] K. Prokes and F. Yokaichiya, JLSRF 3, A104 (2017).

[18] J. P. Perdew, K. Burke, and M. Ernzerhof, Phys. Rev. Lett. 77, 3865 (1996).

[19] G. Kresse and J. Furthmüller, Phys. Rev. B 54, 11169 (1996).

[20] See Supplemental Material at http://link.aps.org/supplemental/ 10.1103/PhysRevB.102.041112 for experimental setup and calculation details.

[21] G. F. Giuliani and A. W. Overhauser, Phys. Rev. B 26, 1660 (1982).

[22] I. A. Zaliznyak, J. M. Tranquada, R. Erwin, and Y. Moritomo, Phys. Rev. B 64, 195117 (2001).

[23] J. A. Wilson, F. J. Di Salvo, and S. Mahajan, Phys. Rev. Lett. 32, 882 (1974). 
[24] S. Kim, K. Kim, and B. I. Min, Sci. Rep. 5, 15052 (2015).

[25] Y. Nagano, N. Araoka, A. Mitsuda, H. Yayama, H. Wada, M. Ichihara, M. Isobe, and Y. Ueda, J. Phys. Soc. Jpn. 82, 064715 (2013).

[26] M. Falkowski, P. Doležal, A. V. Andreev, E. DuvergerNédellec, and L. Havela, Phys. Rev. B 100, 064103 (2019).

[27] K. Ishizaka, T. Arima, Y. Murakami, R. Kajimoto, H. Yoshizawa, N. Nagaosa, and Y. Tokura, Phys. Rev. Lett. 92, 196404 (2004).

[28] H. Miao, R. Fumagalli, M. Rossi, J. Lorenzana, G. Seibold, F. Yakhou-Harris, K. Kummer, N. B. Brookes, G. D. Gu, L. Braicovich, G. Ghiringhelli, and M. P. M. Dean, Phys. Rev. X 9, 031042 (2019).

[29] H. Amitsuka, T. Sakakibara, K. Sugiyama, T. Ikeda, Y. Miyako, M. Date, and A. Yamagishi, Physica B 177, 173 (1992).

[30] See Supplemental Material at http://link.aps.org/supplemental/ 10.1103/PhysRevB.102.041112 for the resistivity and susceptibility measurements of $\mathrm{UPt}_{2} \mathrm{Si}_{2}$.

[31] Z. Hossain, M. Schmidt, W. Schnelle, H. S. Jeevan, C. Geibel, S. Ramakrishnan, J. A. Mydosh, and Y. Grin, Phys. Rev. B 71, 060406(R) (2005).

[32] S. Barua, M. C. Hatnean, M. R. Lees, and G. Balakrishnan, Sci. Rep. 7, 10964 (2017).
[33] H. Amitsuka, M. Sato, N. Metoki, M. Yokoyama, K. Kuwahara, T. Sakakibara, H. Morimoto, S. Kawarazaki, Y. Miyako, and J. A. Mydosh, Phys. Rev. Lett. 83, 5114 (1999).

[34] W. Knafo et al., Nat. Commun. 7, 13075 (2016).

[35] N. Johannsen, S. Süllow, A. V. Sologubenko, T. Lorenz, and J. A. Mydosh, Phys. Rev. B 78, 121103(R) (2008).

[36] See Supplemental Material at http://link.aps.org/supplemental/ 10.1103/PhysRevB.102.041112 for the comparison of $\mathrm{URu}_{2} \mathrm{Si}_{2}$ and $\mathrm{UPt}_{2} \mathrm{Si}_{2}$.

[37] See Supplemental Material at http://link.aps.org/supplemental/ 10.1103/PhysRevB.102.041112 for a discussion on the analogy of ground-state wave vectors of $\mathrm{URu}_{2} \mathrm{Si}_{2}$ and $\mathrm{UPt}_{2} \mathrm{Si}_{2}$.

[38] C. Broholm, J. K. Kjems, W. J. L. Buyers, P. Matthews, T. T. M. Palstra, A. A. Menovsky, and J. A. Mydosh, Phys. Rev. Lett. 58, 1467 (1987).

[39] C. R. Wiebe, J. A. Janik, G. J. MacDougall, G. M. Luke, J. D. Garrett, H. D. Zhou, Y.-J. Jo, L. Balicas, Y. Qiu, J. R. D. Copley, Z. Yamani, and W. J. L. Buyers, Nat. Phys. 3, 96 (2007).

[40] E. Herrera, V. Barrena, I. Guillamón, J. Augusto Galvis, W. J. Herrera, J. Castilla, D. Aoki, J. Flouquet, and H. Suderow, arXiv:2003.07881.

Correction: A clarification has been made to a sentence pertaining to the critical exponent $\beta$ in the paragraph discussing Fig. 3(a). 